\title{
Energy Efficient Ad-hoc Routing Protocol for Disaster Scenario
}

\author{
Vishal M. Patel \\ Student \\ G.H.Patel college of Engineering and Technology \\ V.V.Nagar, Anand Gujarat-India
}

\author{
Nikhil N. Gondaliya \\ Head of Information Technology Department \\ G.H.Patel college of Engineering and Technology \\ V.V.Nagar, Anand Gujarat-India
}

\begin{abstract}
Due to decentralization and infrastructure less characteristics of ad hoc networks, it can be established in any environment without pre-existing infrastructure with ease of deployment. There are many issues for designing of ad hoc network like MAC layer protocol, security, mobility of nodes, routing protocol, quality of service etc... The most challenging is design of routing protocol which may affects the performance of ad hoc network. Ad-hoc networks are very useful in disaster scenario which provides communication among rescue team members to perform relief operation efficiently. In this paper, we have proposed a new technique based on link stability and energy aware, which is applied to Ad-hoc On Demand Distance Vector (AODV) protocol. We have checked performance of normal AODV, Min max based AODV, distance based AODV and our modified AODV routing protocol in terms of routing metrics like packet delivery ratio, normalized routing load, throughput and remaining energy using NS2 simulator. Nodes in ad hoc network are having limited battery power so; if they consume battery power very efficiently then life time of nodes can be increased. From the simulation results, it is observed that our proposed technique on AODV protocol consume less energy and gives better performance than different variant of AODV.
\end{abstract}

\section{Keywords}

MANETs, mobility model, disaster scenarios, and routing protocols

\section{INTRODUCTION}

The mobile ad hoc networks (MANETs) are wireless networks which are deployed without the need of infrastructure. In MANETs each node acts a host and route so the nodes are responsible for routing information from source nodes to the destination nodes. However, nodes in MANETs have several restrictions such as limited coverage area, limited lifetime, memory, and cost [1]. The mobile ad hoc networks is used where there is no communication infrastructure or in disaster search and rescue operations where a network connection is instantly required. The rescue teams can use a Mobile Ad-hoc Network in that situation without requiring a fixed infrastructure. Typically applications covered by MANETs are [1]: search-and-rescue in disaster situations, defence (army, navy, and air force), health care, academic environment, industrial or corporate environment, home network, and sensor network. In this paper, we focus on the use of MANETs in disaster scenarios. Ad hoc networks are oriented towards personal communications and the loss of connectivity to any node is significant. In disaster recovery scenario, it is important that the rescuers do not lose connectivity with any other member of their team, and the connectivity among rescuers should be maintained as long as possible, or at least the duration of the rescue operation. Node send a data packet with some power to the next node and next node receive packet, check the receive power of packet and decode data packet if the power is greater than the default threshold receive power. Furthermore, a node's power available for transmission determines its coverage area and this parameter affects the temporality of the communication links.

Routing protocol implemented at the nodes play an important role on the performance of MANETs. In the case of disaster scenarios the movements of nodes emulate the movements of ambulances taking injured people and other vehicles which take part in rescue operations such as fire engines. Due to the mobility of nodes, the establish communication links are also likely to break frequently. Routing protocols are responsible for reacting whenever a communication link is broken. Depending on the routing protocol, the decision could be to repair the route or to find an alternative or better route to reach the destination.

The mobility models determine how the nodes move in the target scenarios. Aschenbruck et al, proposed a mobility model for disaster scenarios [3]. In this mobility model, the movements of the whole rescue team are modelled. The disaster scenario is divided into different action areas, and the nodes emulate both mobile and static components of the rescue team, such as ambulances or fire fighters. It was included in the mobility generator BonnMotion developed at the University of Bonn (Germany) [4]. It provides an output file which can be integrated in Network Simulator 2 (NS-2) in order to run the simulations. Consequently, the performance of ad hoc network under disaster area scenarios can be analyzed.

To summarize, this paper is organized in 7 sections where Section 2 describes related work in disaster mobility model.IN section 3 describes the disaster area mobility model. In section 4 our proposed technique. The performance evaluation requires scenario of simulation environment setup is presented in Section 5. In Section 6 discussed result analysis and performance evaluation. Finally, In Section 7 describes conclusion and future work.

\section{RELETAED RESEARCH}

Many energy efficient metrics like MTPR(Minimum Transmission Power Routing),MBCR(Minimum Battery Cost Routing),MMBCR(Minimum Maximum Battery Cost Routing),CMMBCR(Conditional Max-Min Battery Capacity Routing) based on this metrics many energy efficient technique proposed by different authors.

In [6], we have checked the performance of the different routing protocols for disaster area. It is observed from the simulation results that ZRP protocol consume less energy than ADOV, DSR, AOMDV protocol, but for another routing metrics like packet delivery ratio, normalized routing load and throughput 
AODV protocol performs better than other protocols like ZRP, DSR and AOMDV.

Suresh Kumar, R K Rathy, D Pandey [1] designed an adhoc network for different disaster recovery scenario and analysed performance of routing metrics with DSDV, DSR, AODV routing protocols. The simulation results reveal that Performance of DSR and AODV is comparable for low mobility and low load scenarios but AODV always performs better than DSR for high load scenarios.

D. G. Reina, S. L. Toral, F. Barrero, N. Bessis [2] evaluated real case scenario in terms of performance using several well-known routing protocols metrics for AODV, DSR and AOMDV routing protocols. Simulations show that AODV provides the best results in terms of routing metrics. DSR and AOMDV could be suitable for those cases where the mobility of nodes is lower.

S.Sridhar et al [7] proposed energy based AODV protocol (ENAODV) announces energy and based on node's packet sending and receiving rates and the sizes of the data to be transmitted. It justifies whether its energy level is maintained or decreased and computes the energy levels of the nodes before selecting path. Utkarsh, Mukesh et al [8] proposed algorithm based on actual distance between the source to destination as well as the minimum available battery power of a node in the path to find the best path for routing.

Sajal Sarkar et al [9] calculated trust of node by own energy factor and energy factor of node to neighbour node as observed by common neighbour node and find the path according to trust. Sofian Hamad et al [10] checked the link expiration time between two nodes when node receive the RREQ message with the predefined threshold and also check the energy of node with predefined threshold and find the better path for routing.

$\mathrm{Xu}$ Zhen et al [11] proposed algorithm based on DSR protocol which divide the energy in to different level (normal, warn and danger) and apply different technique based on energy level.

Jihen et al [12] select the path based on the residual energy, queue length, processing and transmission time of intermediate nodes.

In [13] authors proposed routing based on bandwidth as an application parameter and minimum energy from the path for selection of the path.

Finally, most of the research works in the literature have addressed the different metrics based on energy to improve the route selection mechanism of the routing protocol.In next section we described what disaster area mobility model is.

\section{DISASTER AREA MOBILITY MODEL}

The disaster area mobility model is based on a method called separation of the room [2]. In this model, the disaster scenario is divided into different areas. These areas are: (1) incident site, (2) casualties treatment area, (3) transport zone, and (4) hospital zone shown in Fig 1.



- Incident site: is the place where the disaster actually happened. In this area there are found affected and injured people as well as fatalities, and the disaster (e.g. fire) has to be minimized

- Casualties' treatment area: The casualty treatment area consists of two places: the patients waiting for treatment area and the casualties clearing station. The patients waiting for treatment area is usually close to the incident site. The people are rescued from danger and wait there for their treatment. Then they are transported to the casualties clearing station, which is still within the disaster area.

- Transport zone: is an area where transport units (ambulance coaches and rescue helicopters) wait in stand-by areas to take these people to hospitals.

- Technical operational command: is the zone where the rescue operations are commanded and it is usually located in the casualties' treatment area

Every person participating in the rescue operation belongs to any of the above areas and they are represented by nodes. For example, fire-fighters take part in the incident site whereas paramedics belong to the casualties' treatment areas in order to first evaluate incoming patients. Note that, this mobility model does not take into consideration mobility of patients, so it only models the mobility of the rescue teams. Within the rescue team, two types of nodes can be distinguished, static and mobile nodes. The mobile nodes are normally either people carrying patients or vehicles transporting patients to other locations. The maximum speeds of the mentioned types of nodes are clearly different. Mobility of people is significantly slower than mobility of vehicles. The vehicles transport people to hospitals and then go back to the disaster.

\section{PROPOSED APPROACH}

In our proposed approach multiple RREQ are accepted at destination node and based on the link expiration time, hop count and energy of the nodes, appropriate route is chosen Below the energy model is explained in great details.

\section{Energy Model}

The energy dissipated in transmitting (Etx) or receiving (Erx) one packet can be calculated as:

$$
\begin{aligned}
& E_{t x}=P_{t x} \times \text { Duration } \\
& E_{r x}=P_{r x} \times \text { Duration }
\end{aligned}
$$

where Duration indicates the transmission duration of the packet. When a transmitter transmits a packet to the next hop node all its neighbours receive this packet even it is anticipated to only one of them. Moreover, each node situated between transmitter range and interference range receives this packet but it cannot decode it. These two problems generate waste of energy. So total consume energy is computed by the following equation and also considering sleep and ideal consumption of the energy.

$$
\operatorname{cost}_{t}(i)=E_{t x}+n E_{r x}+E_{s}+E_{i}
$$

\section{Link Expiration Time}

A method to predict the link and the route lifetime based on the nodes' location and movement information between two node $\mathrm{i}\left(\mathrm{x}_{\mathrm{i}}, \mathrm{y}_{\mathrm{i}}\right)$ and $\mathrm{j}\left(\mathrm{x}_{\mathrm{j}}, \mathrm{y}_{\mathrm{j}}\right)$ is given by Link Expiration time[10]

$$
L E T=\frac{-(a+b)+\sqrt{\left(a^{2}+c^{2}\right) r^{2}-(a d-b c)}}{\left(a^{2}+c^{2}\right)}
$$




\section{Energy Efficient Route Discovery Process}

In traditional AODV routing protocol, the source node $S$ sends RREQ and waits for RREP from the destination node D. As the destination node gets the first RREQ, it sends back the RREP through that path as that path is considered as the shortest path. Then after any RREQ received by the destination is discarded. Considering the energy impact on the routing path, it is understood that as the same ath is used for packet transmission by the source and destination node, the energy consumed by the nodes in that path is very high.

This proposed scheme based on number of hop count from source to destination, minimum energy of path, total transmission energy in the path and link expiration time between nodes.

\section{Example:}

As shown in fig. 2, source node 1 send route request to destination node 9 with help of intermediate nodes. Intermediate node receive the RREQ from previous node and compute the link expiration time and check LET with predefine Threshold if it is greater than threshold than forward the RREQ to the next node else drop the RREQ. In this example when node 3 receive RREQ it check with predefine LET threshold $(=0.2)$ so node 3 can not forward the RREQ to the next node.

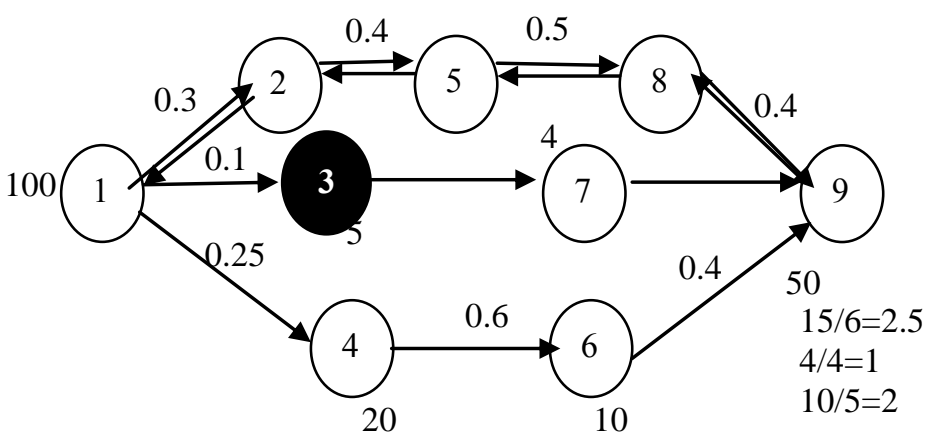

Figure 2: Example of Proposed scheme

In second step to choose best path from source to destination source node send the RREQ packet with two new fields rq_min_energy and rq_power to find minimum energy from the path and total transmission power from source to destination. when the destination node receives the first RREQ, it waits for a $\delta \boldsymbol{t}$ time period to collect other RREQ during that period or maximum RREQ from the source node. After the expiry of the $\boldsymbol{\delta} \boldsymbol{t}$ time period, a best path is selected by computing the cost of each path stored for packet routing.

$$
\text { Cost }=\frac{\text { Minimum residual Energy }}{\text { Total transmission Power }}
$$

This metric consider both remaining energy and transmission power consider if transmission power less than it gives higher ratio and select the route which has higher ratio for unicast route reply.

Below is the algorithm of our proposed technique:

\section{At the Source Node}

When a source node wants to communicate with destination node then source check route cache

1. if (route from source to destination found)

2. $\quad$ i

3. Send RREP to source

4. \} else
It has no route in its cache then it initiates route discovery process and broadcast RREQ packet to its neighbours. Three new information rq_min_energy ,rq_hop_count and rq_trans_power.

total power

added to find minimum energy, hop count and

rq_min_energy : for finding min energy from the path

rq_trans_energy: for total transmission energy of node

\section{At Each Intermediate Node}

Any intermediate node receives the RREQ from neighbour node it will calculate link expiration time and compare with LET_threshold and calculate its remaining energy and compare with minimum energy in RREQ packet. Also calculate hop count to the destination node



5. Else \{

6. Forward RREQ to the next node and increase Hop Count value by 1 and rebroadcast RREQ to its neighbours.

7. $\}$

8. if (rq_min_energy $>$ Current node energy)

$9 . \quad$.

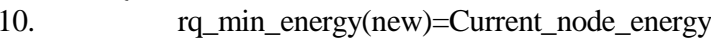

11.

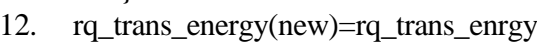

+ trans_energy(current)

\section{At Destination Node}

When a destination node receives RREQ it waits for a $\boldsymbol{\delta} \boldsymbol{t}$ time period to receive RREQ during that period or first three RREQ from the source node. The destination node will receive a RREQ when LET between two node greater than the LET_thrshold. Destination node selects the best path based on the rq_min_energy from different path. The rq_min_energy from different path divide in to three category normal, warn, danger based percentage remaining energy. If more than one RREQ at the destination node in normal than select RREQ which has min hop count and neglect other RREQ which is not in normal. Same as for warn level but in this select RREQ which has max cost rq_min_energy/rq_tran_power and neglect the RREQ if it is in danger.

The pseudo code at destination node is as follows:

1. If it is the first RREQ from the source

2. Setup the Timer for dT duration

3. Wait for other RREQ until dT times passes.

4. $\quad$ End

5. Do the corresponding entry in Seen Table

6. If Time Interval dT passes

7.

8. Send RREP

Calculate best path based on rq_min_energy.

$9 . \quad$ End

10. Divide rq min energy of rreq in three level if it is $>50 \%=$ EN_normal Between $10 \%$ to $50 \%=\mathrm{EN} \_$warn ,$<10 \%=\mathrm{EN}$ danger

11. If value is in EN_normal

$12 . \quad$ Shortest hop route is selected.

13. neglect RREQ which is not in Enormal.

14. Else value is in EN_warn 
15. select RREQ as Max Cost=Rq_min_Energy/Rq_trans_energy from route request

16.

17.

18.

//neglect RREQ which is not in Edanger

Else If value is in Edanger these RREQ can't selected to reply.

From above algorithm, destination node receive RREQ which has link expiration time greater than LET_threshold and save the energy because it's intermediate node can not forward the RREQ and make AODV protocol energy efficient by route selection method describe above. Wait time at destination node can be define as a

Wait Time $=0.5 *($ Residual Energy of dest node / initial energy of node)

In next section describes the scenario for disaster area situation.

\section{MODELING DISASTER SCENARIOS}

We have considered disaster scenarios shown as shown in Fig 1.This scenario evaluated under different routing protocols in our simulation. The size of scenario is $370 \times 220 \mathrm{~m}$. The description of all area is given in Table I.

TABLE 1

SCENARIO

\begin{tabular}{|l|l|l|}
\hline Area & \multicolumn{1}{|c|}{ Size in m } & \multicolumn{1}{|c|}{$\begin{array}{c}\text { Number of } \\
\text { nodes }\end{array}$} \\
\hline Incident Location & $100 \times 100$ & 5 transport \\
\hline Patient waiting zone & $\begin{array}{l}50 \times 40 \text { with } \\
2 \text { zone }\end{array}$ & $\begin{array}{l}5 \text { static, } \\
\text { transport }\end{array}$ \\
\hline $\begin{array}{l}\text { Causalities clearing } \\
\text { Station }\end{array}$ & $\begin{array}{l}75 \times 45 \text { with } \\
3 \text { station }\end{array}$ & 10 transport \\
\hline Ambulances parking point & $25 \times 25$ & $\begin{array}{l}5 \text { static, 10 } \\
\text { transport }\end{array}$ \\
\hline $\begin{array}{l}\text { Technical operational } \\
\text { command }\end{array}$ & $25 \times 50$ & 3 static \\
\hline
\end{tabular}

The numbers of CBR (Traffics) connections among nodes are 25, 30, 40 and 50. These connections are established among nodes of the same area. The total number of connections is divided into 5 groups; each group represents a disaster scenario's areas and send data with one group to another group. The main simulation parameters can be found in Table 2 .

TABLE 2

SIMULATION PARAMETERS

\begin{tabular}{|c|c|}
\hline Parameters & Values \\
\hline NS-2 Version & NS-2.35 \\
\hline Bit rate & $2 \mathrm{Mbps}$ \\
\hline Protocol & AODV,DSR,ZRP, AODV \\
\hline Traffic pattern & Constant Bit Rate (CBR) \\
\hline MAC Protocol & 802.11 \\
\hline Transport protocol & User Datagram Protocol (UDP) \\
\hline Simulation time & $83,103,123$ \\
\hline Number of nodes & $25,30,40,50$ \\
\hline $\mathrm{N}^{\circ}$ connections & 512 bytes \\
\hline Packet size & 4 Pkts/Sec \\
\hline Packet generation rate & $914 \mathrm{MHz}$ \\
\hline Carrier frequency & Vehicles \\
\hline Nodes' speed & $30 \mathrm{~m}$ \\
\hline Transmission range & Two-ray ground \\
\hline Propagation model & \\
\hline
\end{tabular}

\begin{tabular}{|c|c|}
\hline Mobility model & Disaster area mobility model [9] \\
\hline Initial energy of node & $1000 \mathrm{~J}$ \\
\hline Transmission power & $1.3 \mathrm{~W}$ \\
\hline Receiving power & $0.90 \mathrm{~W}$ \\
\hline
\end{tabular}

The following metrics are considered to compare the performance of the routing protocols.

- Throughput: total data packets received successfully by their destinations divided by the simulation time.

- Packet Delivery Ratio (PDR): PDR is the ratio between the number of packets originated by the application layer sources and the number of packets received by the destinations

- Normalized Routing Load (NRL): The number of routing packets transmitted per data packet delivered at the destination

- Average Remaining Energy of Node: It is calculated as the total remaining energy of all node divided by total number of nodes in the network.

\section{SIMULATION ANALYSIS AND RESULTS}

The performance of variants of AODV routing protocol is compared with respect to packet delivery ratio, throughput, normalized routing load and average remaining energy of node. We have checked simulation result of proposed scheme with normal AODV, min max based AODV (OADV-MAX) , distance based AODV (AODV-DIS) and with our proposed technique AODV-PA.

From the Fig. 3, 4, $5 \&$ 6, it is depict that packet delivery ratio, throughput and average remaining energy of node in proposed energy efficient AODV-PA model increased compared to different AODV models. So it clearly indicates that energy is saved in proposed energy efficient AODV. While normalized routing load is decreased in proposed energy efficient AODV model than other AODV models. So it indicate less routing load because no need to send more routing packet for communication. The below result are taken for varied number of nodes.

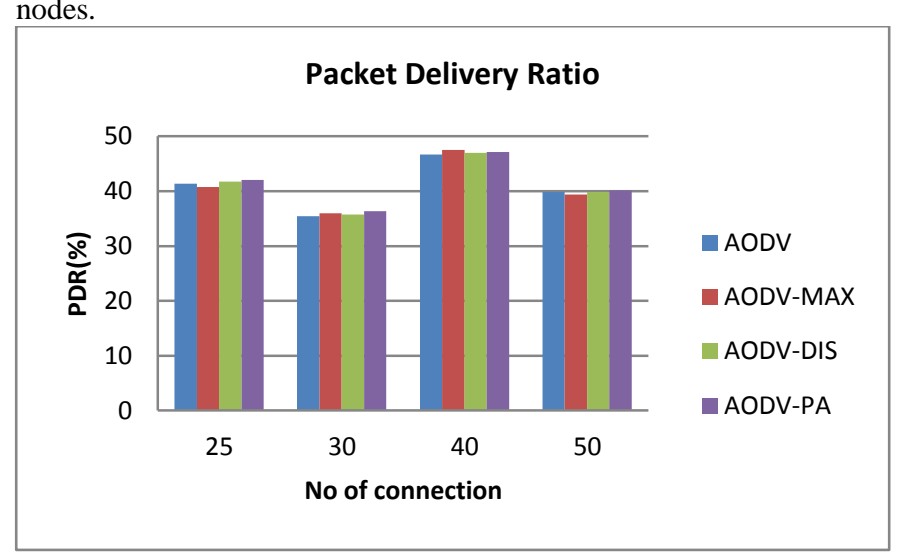

Figure 3. Packet delivery ratio for 83 node

Analysis 1: PDR for 103 and 123 node for scenario-I

Figures 7 and 8 shows the simulation result of PDR for 103 and 123 nodes for AODV models. It is depicted that packet delivery ratio in proposed energy efficient AODV-PA model increased compared to different AODV models. 


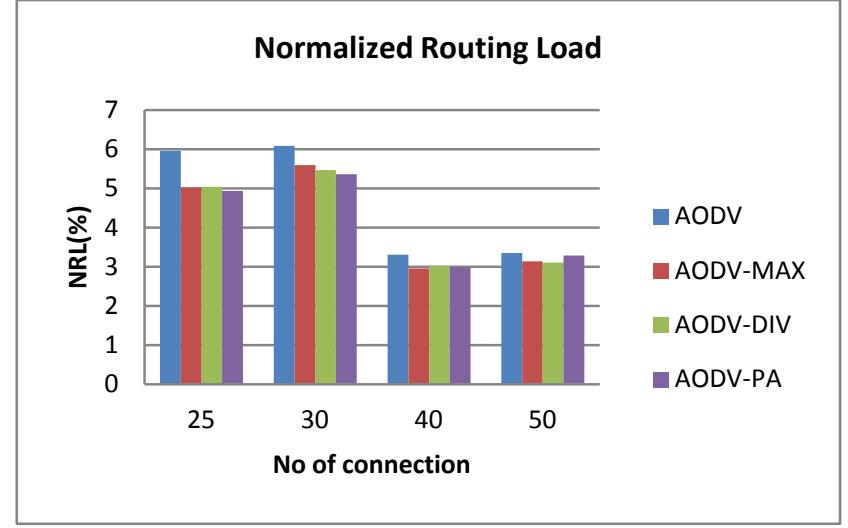

Figure 4 Normalized Routing Load for 83 node

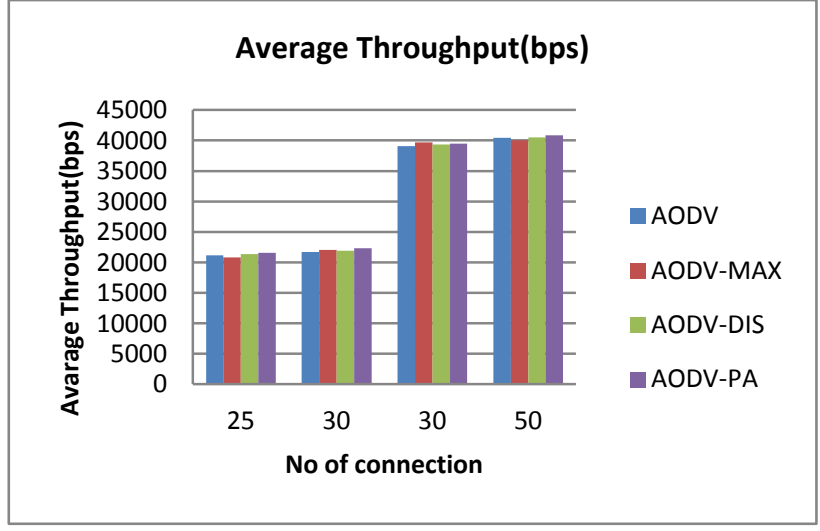

Figure 5 Average Throughput for 83 node

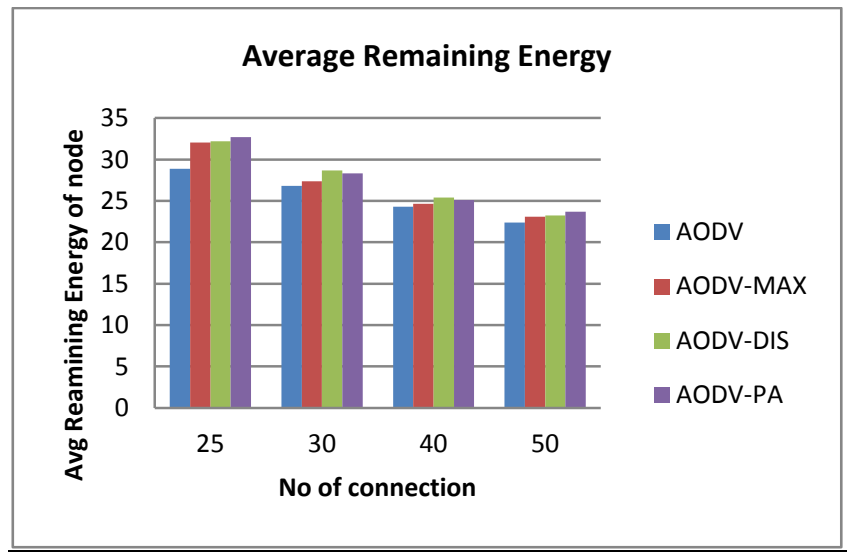

Figure 6 Average remaining energy of node for 83 node

Fig 3 shows that for AODV-MAX the PDR is decreased than other AODV models it indicates that AODV-MAX dose not perform better performance in all condition.

\section{Analysis 2: NRL for 103 and 123 node for scenario-I}

From the Fig. $9 \&$ 10, it is depicted that NRL in proposed energy efficient AODV-PA model is decreased compared to different AODV models. All the AODV models give better performance than traditional AODV

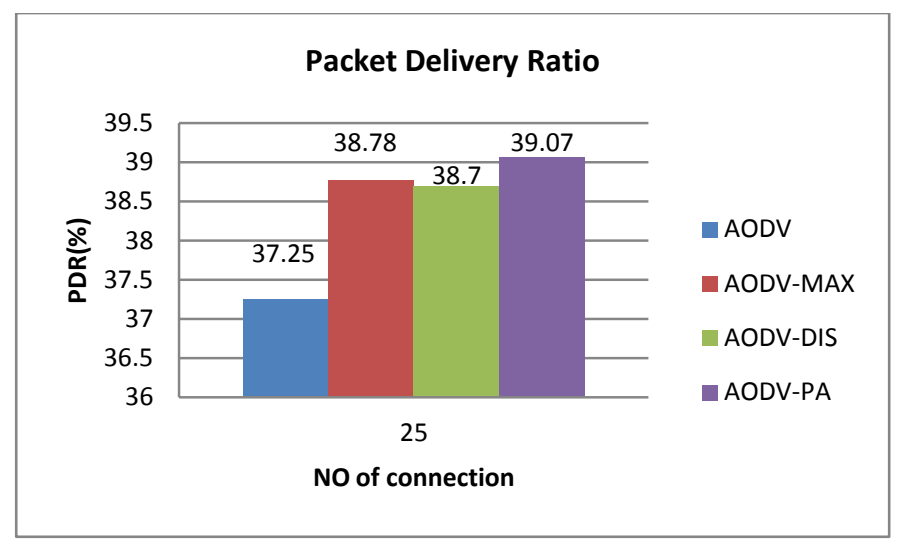

Figure 7. Packet delivery ratio for 103 node

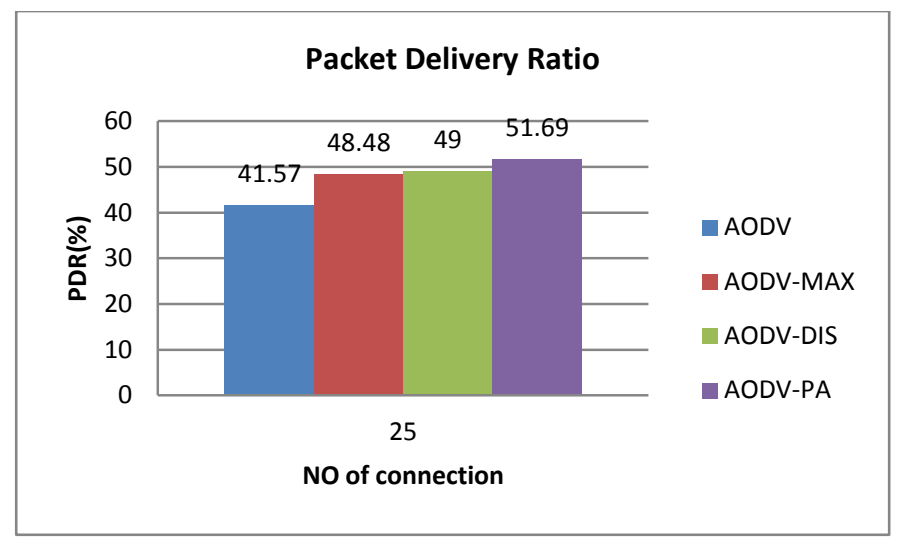

Figure 8. Packet delivery ratio for 123 node

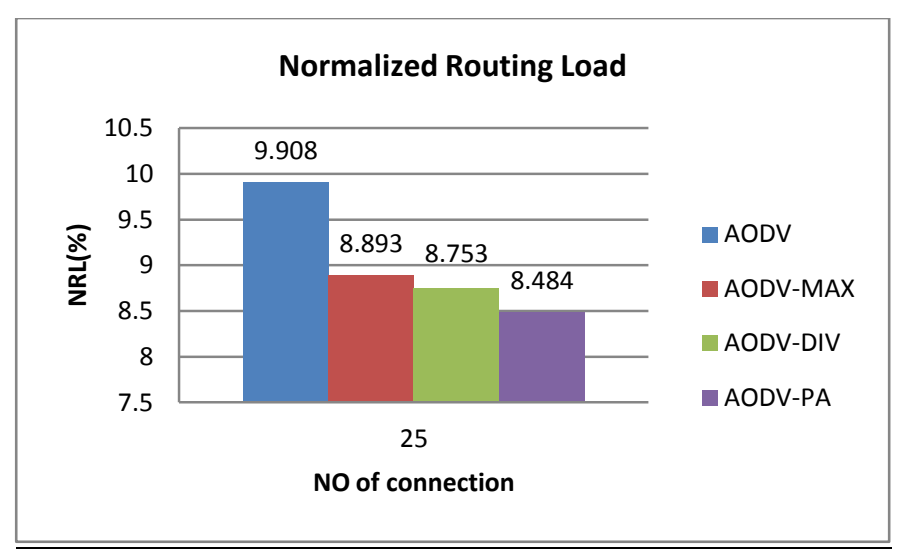

Figure 9 Normalized Routing Load for 103 node

Analysis 3: Average throughput for 103 and123 node for Scenario-I

From the Fig. $11 \& 12$, it is observed that throughput in proposed energy efficient AODV-PA model increased compared to different AODV models. All the AODV models give better performance than traditional AODV. AODV-MAX does not give better performance in all condition. 




Figure 10 Normalized Routing Load for 123 node

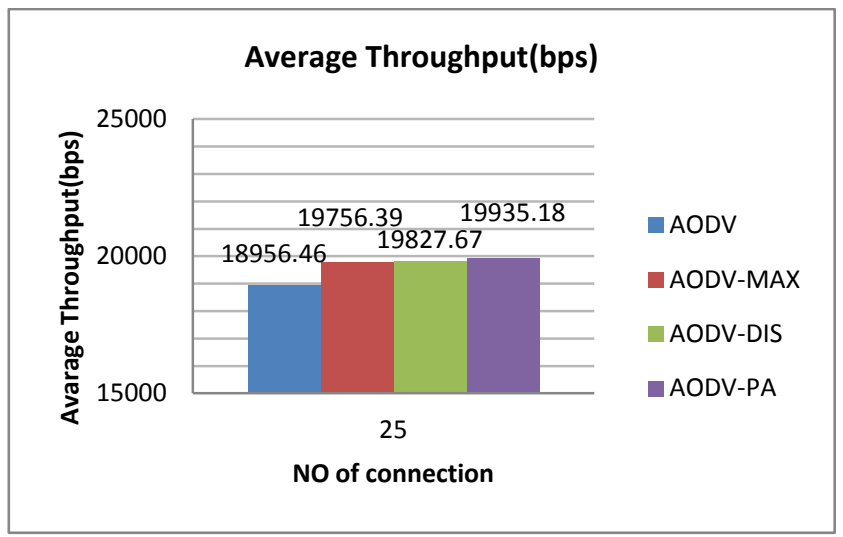

Figure 11 Average Throughput for 103 node



Figure 12 Average Throughput for 123 node

Analysis 4: Average Remaining energy for 83,103 and 123 node for scenario-I

From the Fig. $13 \& 14$, it is observed that remaining energy of the node in proposed energy efficient AODV-PA model increased compared to different AODV models. All the AODV models give better performance than traditional AODV. AODV-PA gives better result than any other AODV models because it finds the path which has better link expiration time due to LET_thresold and applying energy saving technique given in proposed scheme.

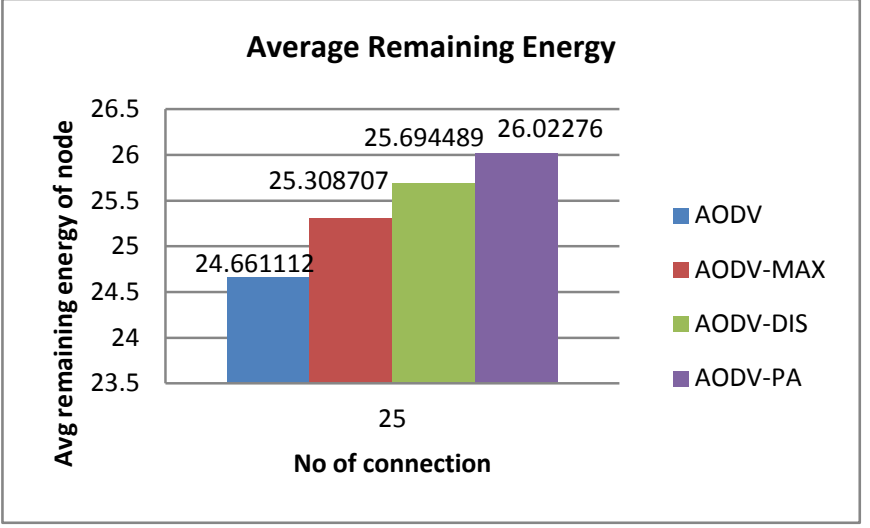

Figure 14 Average remaining energy of node for 103 node

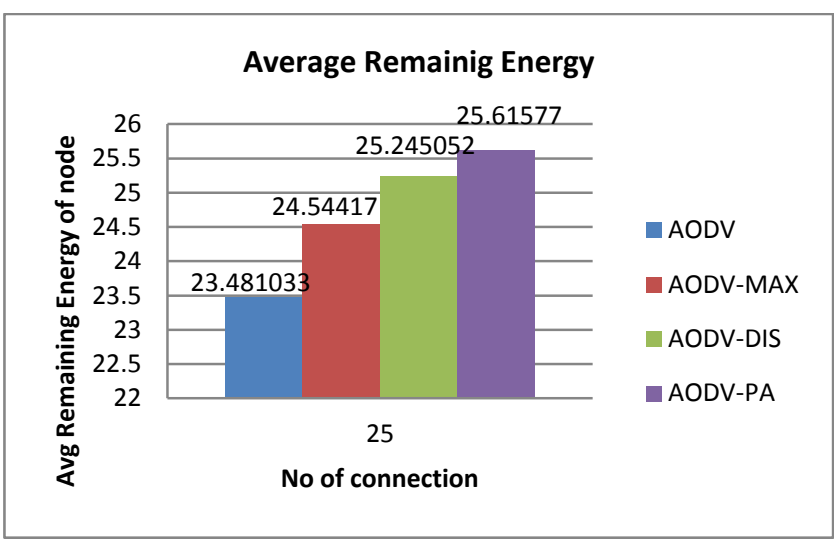

Figure 14 Average remaining energy of node for 123 node

\section{CONCLUSION AND FUTUREWORK}

Our proposed technique provides better energy efficient routing in post disaster area. The simulation results show that our proposed technique improves the PDR, throughput and lessen the NRL and energy consumption in the network in the network. In future we will improve this proposed solution to acquire much better path stability. Here we have applied link expiration time threshold is predefined we can apply some another technique to find the stability and also use some another technique to find the trusted path at destination node.

\section{ACKNOWLEDGEMENT}

My Sincere thanks to my guide Prof. Nikhil N Gondaliya, for providing me an opportunity to do my research work. I express my thanks to my institute namely G. H. Patel College of Engineering and Technology for providing me with a good environment and facilities like Internet, books, computers and all that as my source to complete this research work. My heart-felt thanks to my family, friends and colleagues who have helped me for the completion of this work..

\section{REFERENCES}

[1] Suresh Kumar, R K Rathy, D Pandey,'Design of an Ad-hoc Network Model for Disaster Recovery Scenario Using Various Routing Protocols", International Conference on Advances in Computing, Communication and Control (ICAC3'09)-2009.

[2] D. G. Reina, S. L. Toral, F. Barrero, N. Bessis, E. Asimakopoulou,"Evaluation of Ad Hoc Networks in 
Disaster Scenarios", Third International Conference on Intelligent Networking and Collaborative Systems-IEEE 2011

[3] N. Aschenbruck, M. Frank, P. Martini, J. Tölle. "Human Mobility in MANET Disaster Area Simulation - A realistic Approach". $29^{\text {th }}$ Annual IEEE International Conference on Local Computer Network (LCN'04). 2004.

[4] N. Aschenbruck, R. Ernst, E. Gerhards-Padilla, M. Schwamborn. "BonnMotion- A mobility scenario generation and Anlysis tool". Simutool -2010

[5] Perkins C., Belding-Royer E., Das S. , Adhoc On-Demand Distance Vector (AODV) Routing, RFC 3561, July 2003.

[6] Vishal M. Patel, Nikhil N. Gondaliya,"Performance Analysis of Ad-hoc Routing Protocols for Disaster Scenario",International Journal of Research in Information Technology- january-2014S.

[7] Sridhara,R.Baskaranb ,P.Chandrasekar ,"Energy supported AODV (EN-AODV) for QoS routing in MANET,", Published by Elsevier 2013.

[8] Utkarsh, Mukesh Mishra2 and Suchismita Chinara," ESAR: An Energy Saving Ad Hoc Routing Algorithm for MANET", IEEE- Fourth International Conference on Advanced Computing, ICoAC 2012

[9] Sajal Sarkar and Raja Datta," A Trust Based Protocol for Energy-Efficient Routing in Self-Organized MANETs",IEEE-2012
[10] Sofian Hamad, Hadi Noureddine and Hamed AlRaweshidy,"LSEA: Link Stability and Energy Aware for Efficient Routing in Mobile AdHoc Network",IEEE-2011

[11] Xu Zhen, Xiao Juan," Energy-aware and Delay-aware QoS Routing in Mobile Ad-Hoc Networks",IEEE-2012

[12] Jihen DRIRA REKIK, Leïla BACCOUCHE, Henda BEN GHÉZALA," Load-balancing and Energy Aware Routing Protocol for real-time flows in Mobile Ad-Hoc Networks",IEEE -2011

[13] Vinay Rishiwal, S. Verma and S. K. Bajpai ,"QoS Based Power Aware Routing in MANEs", International Journal of Computer Theory and Engineering, April- 2009

[14] Teerawat Issariyakul, Ekram Hossain, "Introduction to Network Simulator NS2”, Springer 2008.

[15] N. Achenbruck, E. Gerhaps-Padilla, M. Gerharz, M. Frank, P. Martini. "Modelling Mobility in Disaster Area Scenarios". In The 10- th ACM International Symposium on Modeling, Analysis and Simulation of Wireless and Mobile Systems

[16] Zussman Gil , Segall Adrian, "Energy Efficient Routing in Ad Hoc Disaster Recovery Networks", IEEE INFOCOM 2003

[17] Network Simulator tutorial www.isi.edu/nsnam/ns/tutorial

[18] Charles E. Perkins, “Ad Hoc Networking”,2008 ISBN 97881-317-20967 